\title{
PILOTING CHANGES TO CHANGING AIRCRAFT DYNAMICS: WHAT DO PILOTS NEED TO KNOW?
}

\author{
Anna C. Trujillo and Dr. Irene Gregory, NASA Langley Research Center, Hampton, VA
}

\begin{abstract}
An experiment was conducted to quantify the effects of changing dynamics on a subject's ability to track a signal in order to eventually model a pilot adapting to changing aircraft dynamics. The data will be used to identify primary aircraft dynamics variables that influence changes in pilot's response and produce a simplified pilot model that incorporates this relationship. Each run incorporated a different set of second-order aircraft dynamics representing short period transfer function pitch attitude response: damping ratio, frequency, gain, zero location, and time delay. The subject's ability to conduct the tracking task was the greatest source of root mean square error tracking variability. As for the aircraft dynamics, the factors that affected the subjects' ability to conduct the tracking were the time delay, frequency, and zero location. In addition to creating a simplified pilot model, the results of the experiment can be utilized in an advisory capacity. A situation awareness/prediction aid based on the pilot behavior and aircraft dynamics may help tailor pilot's inputs more quickly so that PIO or an upset condition can be avoided.
\end{abstract}

\section{Introduction}

Significant research has been conducted to model or identify the pilot, as a way to quantify handling qualities or to better understand the behavior of a human pilot in controlling a vehicle [17]. Our research is investigating new analytical methods to model the pilot's changing behavior over short time periods in response to changing aircraft dynamics.

A model that captures the pilot's adaptability to changing aircraft dynamics without a priori knowledge of these changes is the long-term objective of this research. Availability of such an analytical model would have impact in a number of different areas; among these are decision aids for the pilot, function allocation between the pilot and automation especially during changes in effective dynamics, and potential requirements for adaptive control law design. The research presented here builds on past work to provide tools to analyze and solve this type of modeling problem.

\section{Method}

An experiment was conducted to quantify the effects of changing dynamics on a subject's ability to track a signal in order to eventually model a pilot adapting to changing aircraft dynamics. This paper documents the development of a database that will be used for this work. The experiment evaluated primary aircraft dynamics variables that influence changes in pilot's response and produce a simplified pilot model that incorporates this relationship.

The experiment, conducted to identify the pilotdynamic variable relationship, required each subject to track as closely as possible a pitch attitude signal. The input for the tracking task was designed to contain a variety of frequencies in the frequency range associated with human pilot inputs, namely 0.1 to $10 \mathrm{rad} / \mathrm{sec}$. Each run incorporated a different set of second-order aircraft dynamics representing short period transfer function pitch attitude response. This response was then displayed to the pilot as the aircraft response.

\section{Independent Variables}

The following independent variables were controlled during each data run.

\section{Pitch Dynamics}

This experiment looked at short period dynamics, which affect flying qualities the most. Therefore, longitudinal dynamics were controlled while lateral/directional and thrust values were held constant at a heading of $40 \mathrm{deg}$ and a speed of 300 kts. The altitude at the beginning of each data run was $10,000 \mathrm{ft}$. The aircraft short-period pitch attitude transfer function was:

$$
\frac{\theta}{\delta}(s)=\frac{K\left(s+L_{\alpha}\right)}{s\left[(s / \omega)^{2}+2 s(s / \omega)+1\right]} e^{-\tau s}
$$


where $\theta$ is pitch attitude and $\delta$ is pilot stick. The damping ratio $(\varsigma)$ was $0.4,0.7$, or 1 . The short period frequency $(\omega)$ was $0.5 \mathrm{~Hz}, 1 \mathrm{~Hz}$, or $1.5 \mathrm{~Hz}$. Gain $(K)$ was 1 , 2, or 3. Zero location $\left(L_{\alpha}\right)$ was $0.5,1$, or 1.5 . Lastly, time delay $\tau$ was $0 \mathrm{~ms}, 75 \mathrm{~ms}$, or $150 \mathrm{~ms}$.

\section{Tracking Signal}

The maneuver was designed as a $30 \mathrm{sec}$ longitudinal tracking task. The tracking command input contained a range of frequencies and amplitudes to excite an appropriate dynamic range.

The longitudinal tracking task was designed to contain a variety of discrete frequencies in the frequency range associated with human pilot inputs, namely 0.1 to $10 \mathrm{rad} / \mathrm{sec}$. The input design method is described in detail by Klein and Morelli $[8,9]$. The result was the equivalent of a frequency sweep input, but used many sinusoidal components with discrete frequencies applied simultaneously, instead of a single sinusoid with frequency increasing monotonically in time.

Figure 1 shows an example tracking task. The sum-of-sines task was generated as:

$$
u(t)=\sum_{k \in\{1,2, \ldots, M\}} A_{k} \sin \left(\frac{2 \pi k t}{T}+\phi_{k}\right)
$$

where $u(t)$ is the pitch attitude tracking command (deg), the discrete frequencies $2 \pi k / T$ were chosen to span the frequency range of 0.1 to $10 \mathrm{rad} / \mathrm{sec}$, the phase angles $\left(\phi_{k}\right)$ were random, and the amplitudes

$\left(A_{k}\right)$ were chosen to customize the power spectrum of the multi-sine input. In this application, the phase angles were chosen at random to emulate a random sinusoidal input. The frequency indices $(k)$ were chosen at irregular intervals, which also helps to emulate a random sinusoidal input.

\section{Subjects}

Four pilots participated as subjects. The average age was 50 (standard deviation of 3.6) years old. The average years flying was $20.5 \pm 12$ and the average number of flight hours was $3312 \pm 4483 \mathrm{hrs}$. Subject 2 had the least number of flight hours $(<600 \mathrm{hrs})$ and years flying $(\approx 6)$ whereas the next closest subject had just about 1000 flight hours and approximately 15 years of flying experience. Subject 4 had the most
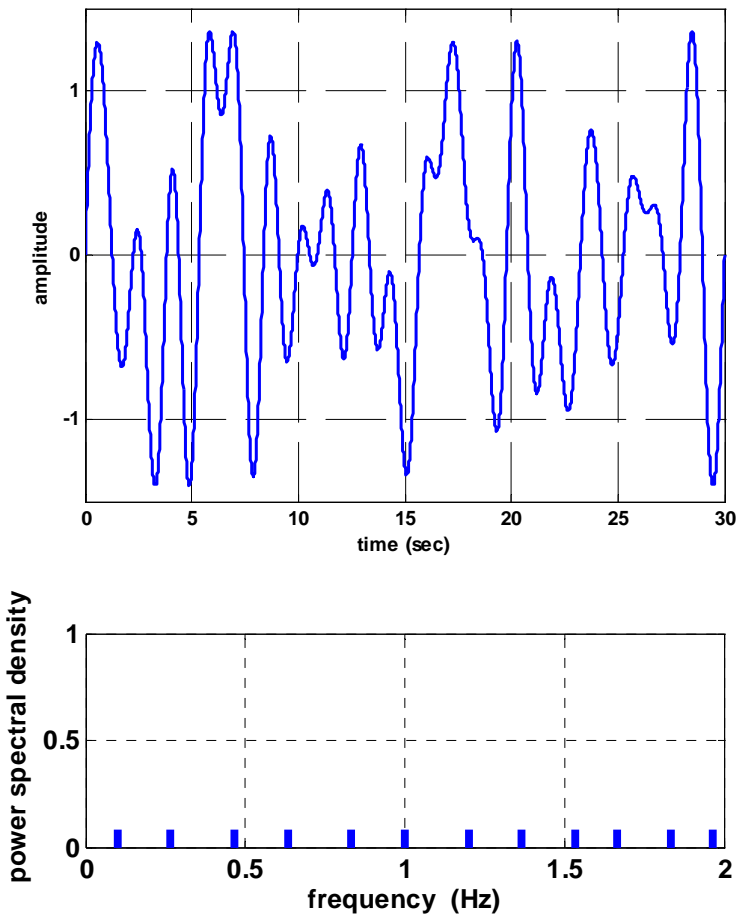

Figure 1. Longitudinal Input Signal

number of flight hours $(>10,000 \mathrm{hrs})$ and the most years flying ( $>30$ years) while the next nearest subject had just under 2000 flight hours.

\section{Dependent Variables}

During each of the 35 data runs, subjects tracked the pitch command input signal as closely as possible for $30 \mathrm{sec}$. After each run, subjects then completed a Cooper-Harper $(\mathrm{CH})$ rating scale.

\section{RMS Error}

Root Mean Square (RMS) error was calculated by taking the current pitch attitude of the aircraft and subtracting the desired pitch attitude of the aircraft. This was calculated in deg.

\section{CH Rating}

At the end of each data run, subjects gave a modified $\mathrm{CH}$ rating $[10,11]$. An electronic format of the $\mathrm{CH}$ rating process was used which enforced the structure of the $\mathrm{CH}$ logic decision path, starting at the bottom ("Is it controllable?") format (Fig. 2), and progressing accordingly until a final rating is given [12]. The pitch attitude command signal $(u)$ drove the flight director symbol on the Attitude Direction Indicator (ADI) shown in Figure 3. Zero tracking error was defined by the airplane symbol nudged 

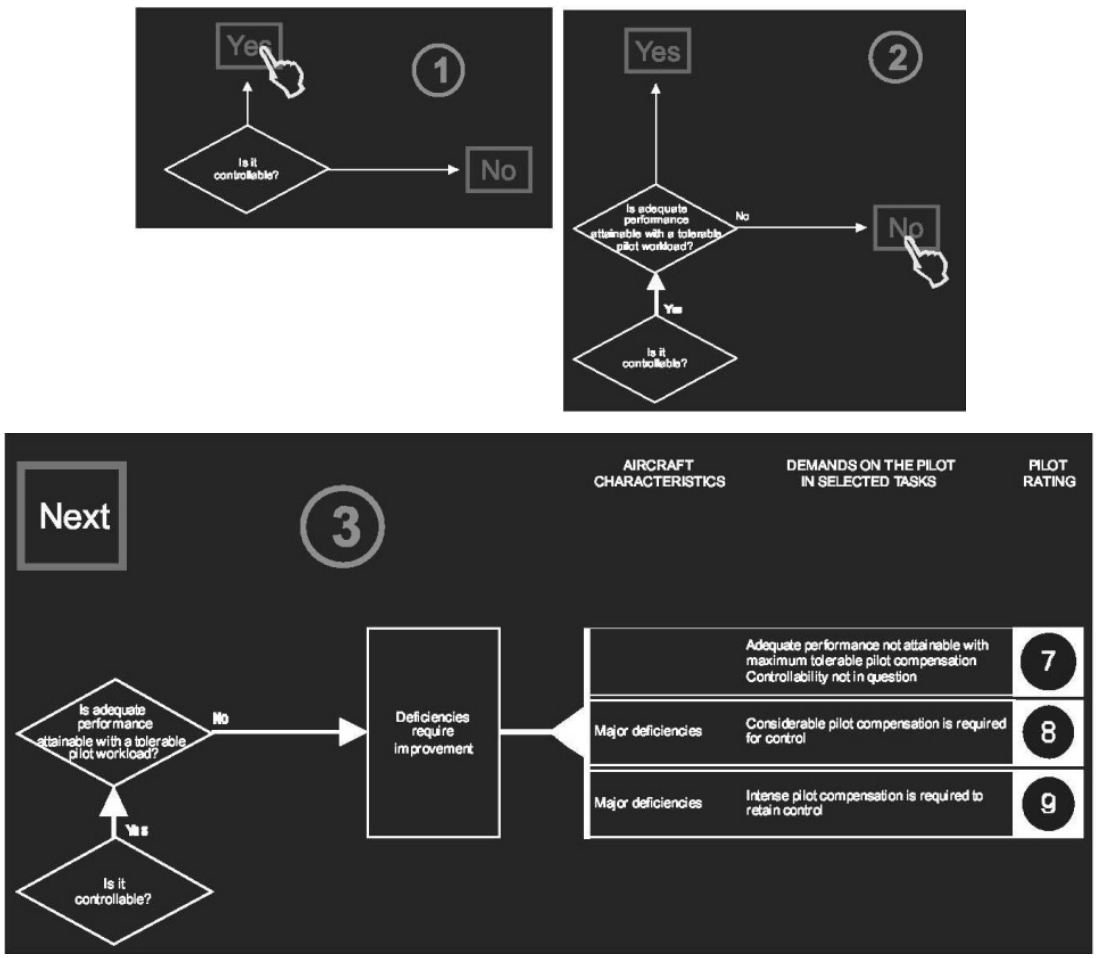

Figure 2. Cooper-Harper Controllability Scale Format

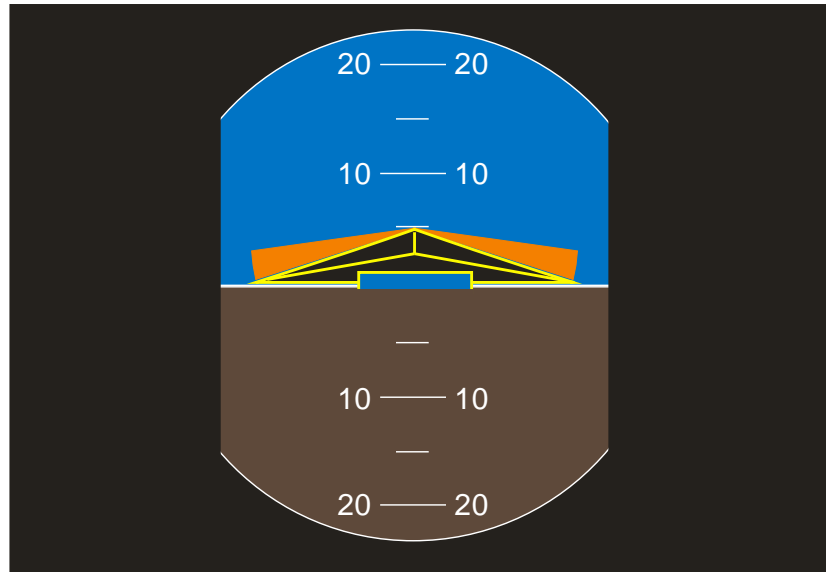

Figure 3. CH Optimal Rating Performance

under the flight director (Fig. 3). Desired performance was defined as being within 2.5 degrees, which was half the width of the airplane symbol wings, of the flight director (Fig. 4). Adequate performance was defined as being within 5 degrees of the flight director which was the width of the airplane symbol wings (Fig. 5).

\section{Procedure}

After arrival, subjects were briefed on the experiment, task, and displays using still pictures.

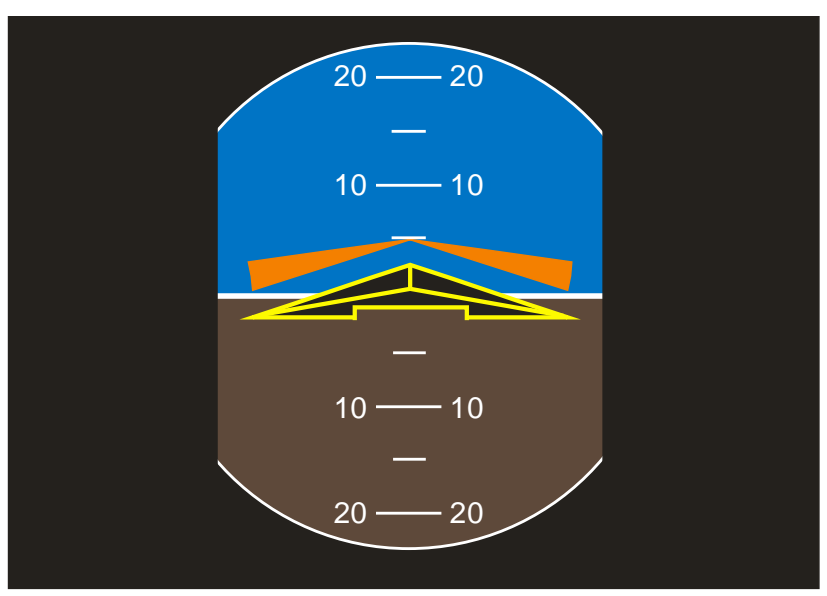

Figure 4. CH Desired Rating Performance

Next, subjects were introduced to the simulator using a 20-minute familiarization run. The familiarization run had a 5 min periodic longitudinal tracking signal similar to the data runs. For both the familiarization run and for the data runs, the flight director had an amplitude of no more than \pm 5 degrees. During the familiarization run, the subject was also able to free fly the simulation. The pitch attitude response transfer function (see Eq. 1) had the baseline characteristics of: $\zeta=0.4, \omega=0.5 \mathrm{~Hz}, \tau=0$ $\mathrm{ms}, \mathrm{L}_{\alpha}=1.5$, and $K=3$. Subjects were allowed to fly 


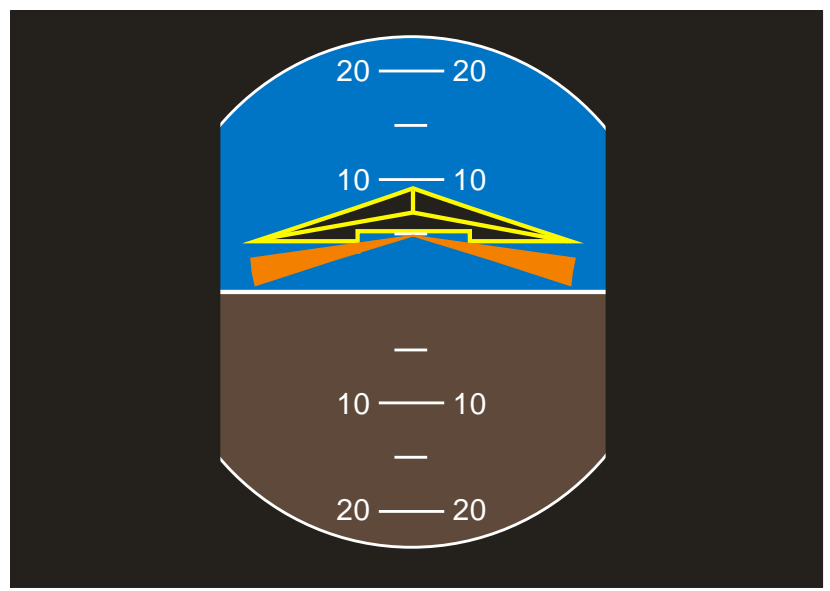

Figure 5. CH Adequate Rating Performance

the familiarization run until they were comfortable with the simulation dynamics.

After the subject was comfortable with the sidestick and the task, each subject completed 35 data runs. During each data run, straight and level flight was established for $2 \mathrm{sec}$ before the tracking task initiated. After the $30 \mathrm{sec}$ tracking task, straight and level flight was reestablished for $2 \mathrm{sec}$. Therefore, each run lasted a total of $34 \mathrm{sec}$. At the end of the runs, subjects gave a $\mathrm{CH}$ rating.

The simulation was implemented in Matlab/Simulink ${ }^{\circledR} 2009$ b. The simulator setup is shown in Figure 6 and the general simulation diagram is shown in Figure 7.

\section{Results}

Analyses were done using SPSS $^{\mathrm{TM}}$ v16. Significance was set at a $p$-value $\leq 0.05$.

\section{Subject Effects on RMS Error}

Individual subjects tracking variability had the greatest effect on RMS error $\left(F_{(3,201)}=11.418\right.$, $\mathrm{p} \leq 0.01)$. These data are shown in Figure 8 as the mean RMS error for each subject collapsed across all

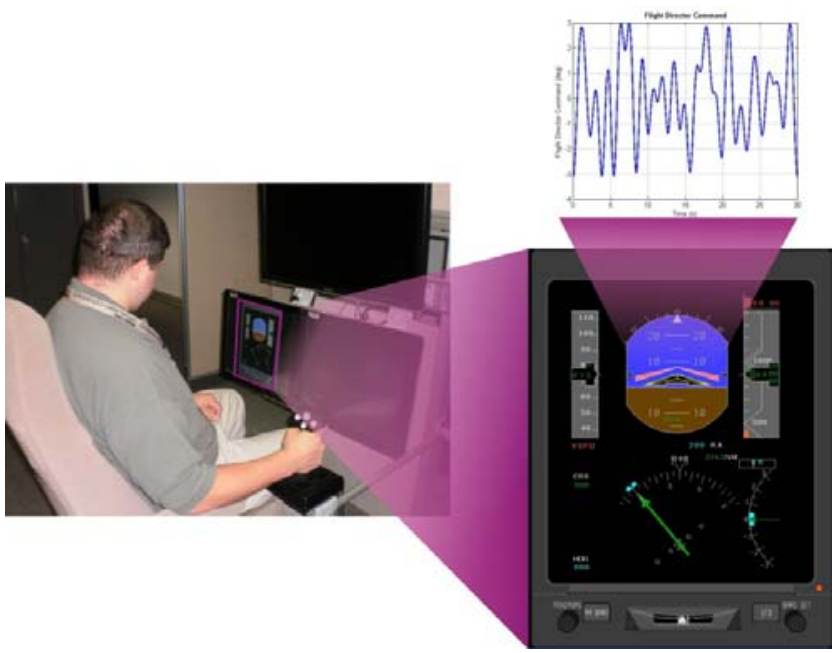

Figure 6. Simulator Setup

aircraft dynamics variations with deviation bars, indicating 1 unit of computed standard error (SE) from the mean. A closer look at the data indicated that the subject with the larger RMS error had less flight hours (Subject 2 flight hours $<600$ ) as compared to the other subjects who had more flight hours and a smaller RMS error.

\section{Aircraft Dynamic Effects on RMS Error}

Tables 1 and 2 detail how the aircraft dynamics affected the RMS error. As seen in Table 1, all individual aircraft dynamics significantly affected the RMS error. The most significant effects were the time delay, frequency, and zero location as indicated by $\eta^{2}$ (Table 1 ).

\section{Time Delay Effects on RMS Error}

For the $150 \mathrm{~ms}$ time delay, subjects' RMS error was significantly larger than for the 0 and $75 \mathrm{~ms}$ time delay (Fig. 9). This increase in RMS error was also reflected by the subjects' $\mathrm{CH}$ rating. As can be seen in Figure 10, as the time delay increased, the vehicle moved towards more Level 2 and 3 handling qualities.

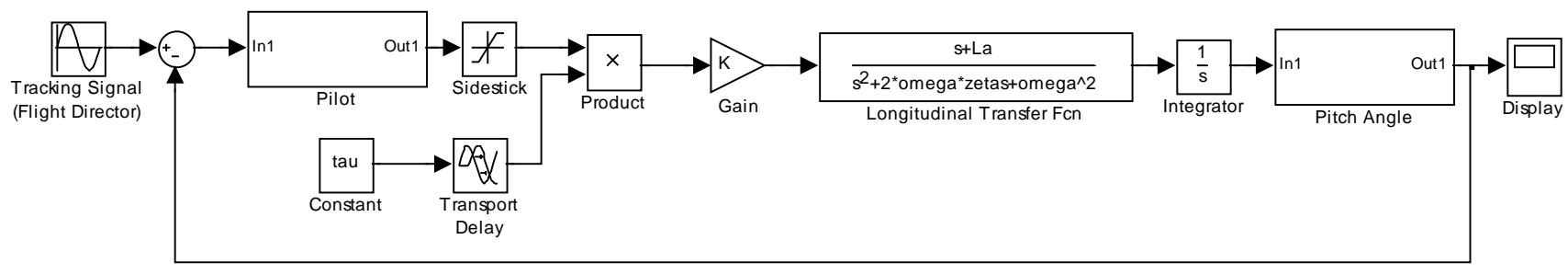

Figure 7. Block Diagram of the Simulation 


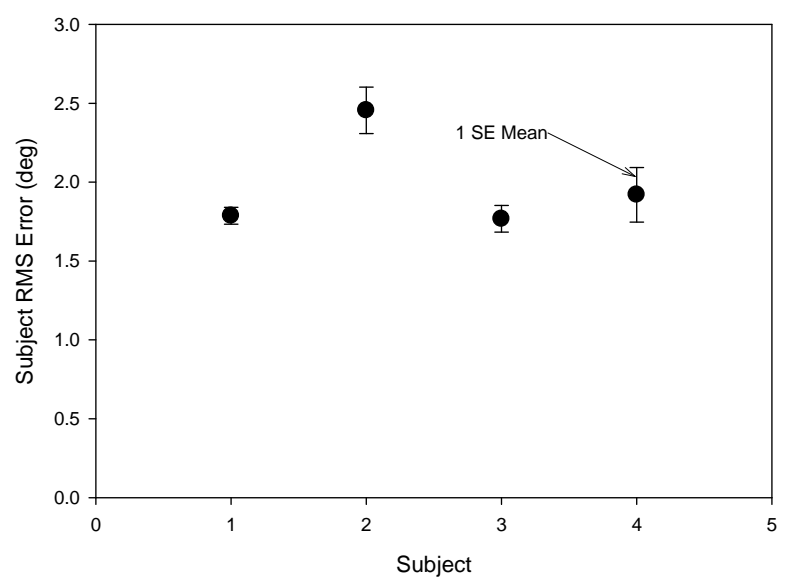

Figure 8. RMS Error by Subject

Table 1. Effects of Dynamics on RMS Error

\begin{tabular}{|l|r|r|c|}
\hline Aircraft Dynamic & $\boldsymbol{F}_{(\mathbf{1 , 2 0 7 )}}$ & $\mathbf{p}$ & $\boldsymbol{\eta}^{\mathbf{2}}$ \\
\hline Gain $[\mathrm{K}]$ & 9.64 & $\leq 0.01$ & 0.023 \\
\hline Zero Location $\left[\mathrm{L}_{\alpha}\right]$ & 26.12 & $\leq 0.01$ & 0.063 \\
\hline Damping Ratio $[\zeta]$ & 6.95 & $\leq 0.01$ & 0.017 \\
\hline Frequency $[\omega]$ & 58.20 & $\leq 0.01$ & 0.141 \\
\hline Time Delay $[\tau]$ & 14.99 & $\leq 0.01$ & 0.036 \\
\hline
\end{tabular}

Table 2. RMS Error by Aircraft Dynamics

\begin{tabular}{|l|c|c|c|}
\hline \multicolumn{2}{|c|}{ Aircraft Dynamic } & $\begin{array}{c}\text { Mean RMS } \\
\text { Error (deg) }\end{array}$ & SE \\
\hline \multirow{2}{*}{ Gain $[\mathrm{K}]$} & 1 & 1.86 & 0.04 \\
\cline { 2 - 4 } & 2 & 1.75 & 0.05 \\
\cline { 2 - 4 } & 3 & 2.40 & 0.17 \\
\hline Zero & 0.5 & 1.74 & 0.35 \\
\cline { 2 - 4 } Location & 1.0 & 1.75 & 0.38 \\
\cline { 2 - 3 }$\left[\mathrm{L}_{\alpha}\right]$ & 1.5 & 2.28 & 1.21 \\
\hline Damping & 0.4 & 2.19 & 0.12 \\
\cline { 2 - 4 } Ratio $[\zeta]$ & 0.7 & 1.70 & 0.05 \\
\cline { 2 - 3 } & 1.0 & 1.90 & 0.05 \\
\hline Frequency & 0.5 & 2.46 & 1.22 \\
\cline { 2 - 3 }$(\mathrm{Hz})[\omega]$ & 1.0 & 1.70 & 0.32 \\
\cline { 2 - 3 } & 1.5 & 1.64 & 0.24 \\
\hline Time & 0 & 1.85 & 0.05 \\
\cline { 2 - 2 } Delay & 75 & 1.70 & 0.05 \\
\cline { 2 - 2 }$(\mathrm{ms})[\tau]$ & 150 & 2.28 & 0.13 \\
\hline
\end{tabular}

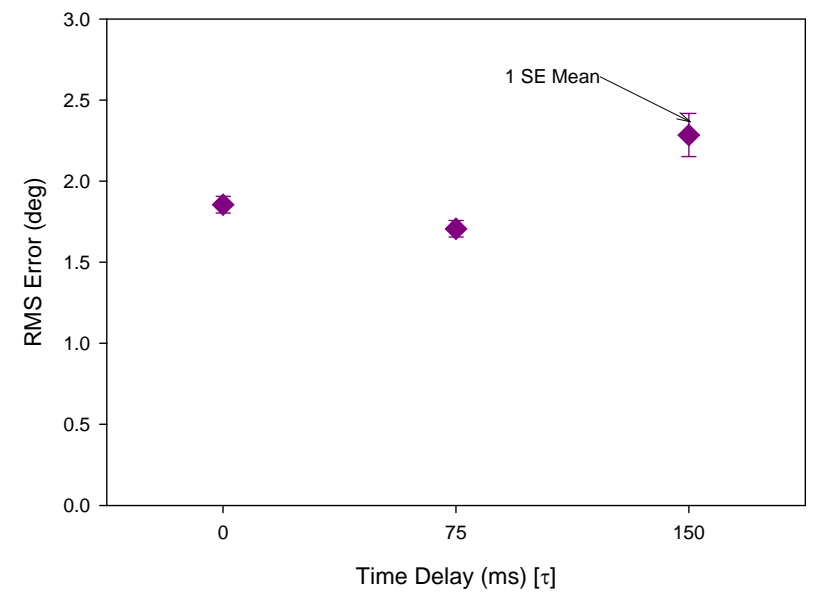

Figure 9. RMS Error by Time Delay

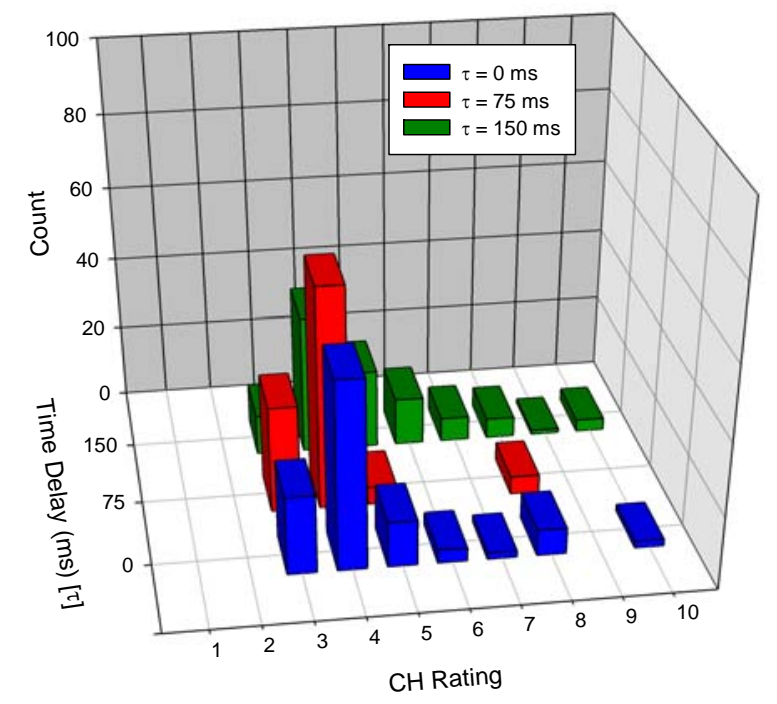

Figure 10. CH Rating Count by Time Delay

\section{Frequency Effects on RMS Error}

The RMS error for the frequency increased as the speed of the system response decreased down to $0.5 \mathrm{~Hz}$ (Fig. 11) which was different from the 1.0 and $1.5 \mathrm{~Hz}$ frequencies. As with the time delay, subjects' $\mathrm{CH}$ ratings also indicated this increase in RMS error (Fig. 12). The handling qualities shifted to Level 2 and 3 as the frequency decreased. 


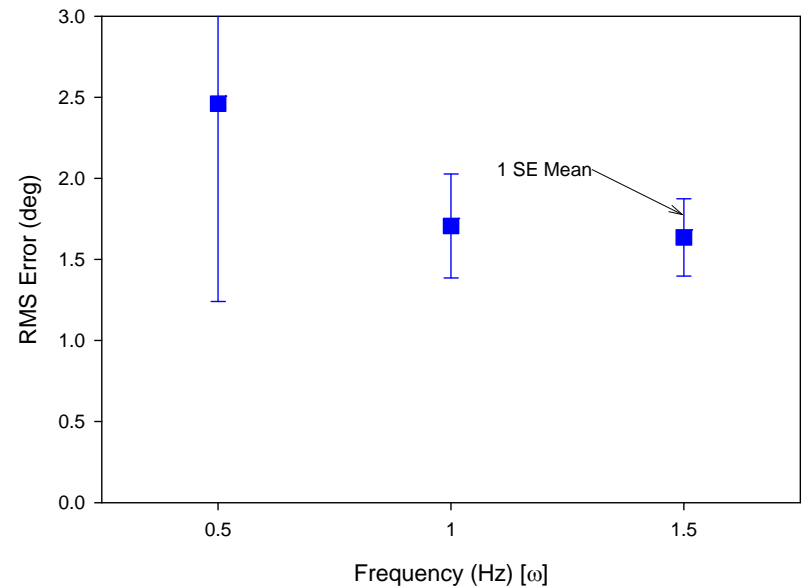

Figure 11. RMS Error by Frequency

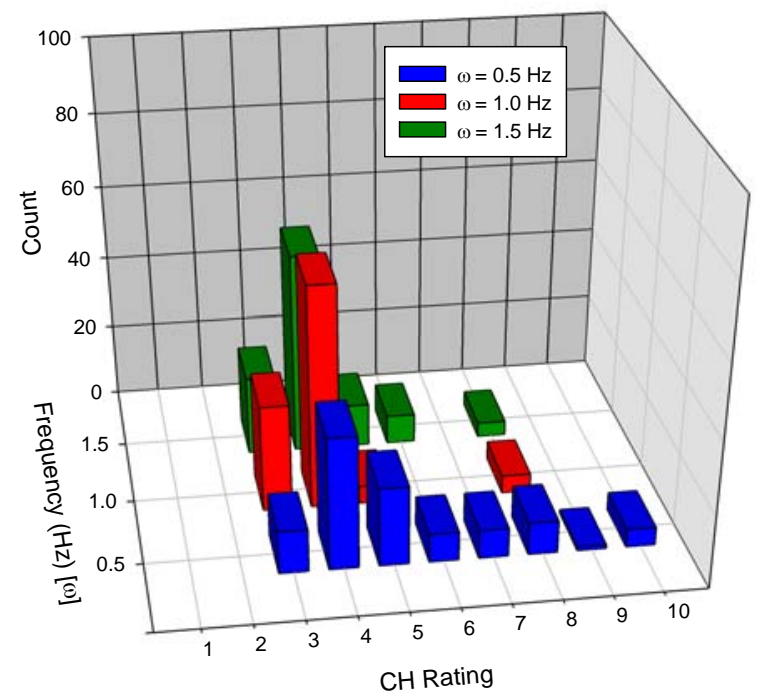

Figure 12. CH Rating Count by Frequency

\section{Zero Location Effects on RMS Error}

Lastly, RMS error increased with a zero location further into the left half plane (Fig. 13). Once again, this increase in RMS error manifests itself in the subjects' $\mathrm{CH}$ ratings. As the zero location moves farther into the left-half plane, the subjects' $\mathrm{CH}$ rating spread increases towards Level 2 and 3 handling qualities (Fig. 14). Furthermore, this increase in RMS error was even more pronounced with a zero location further into the left half plane for lower frequencies (Fig. 15) as would be expected by the control anticipation parameter (CAP) which is

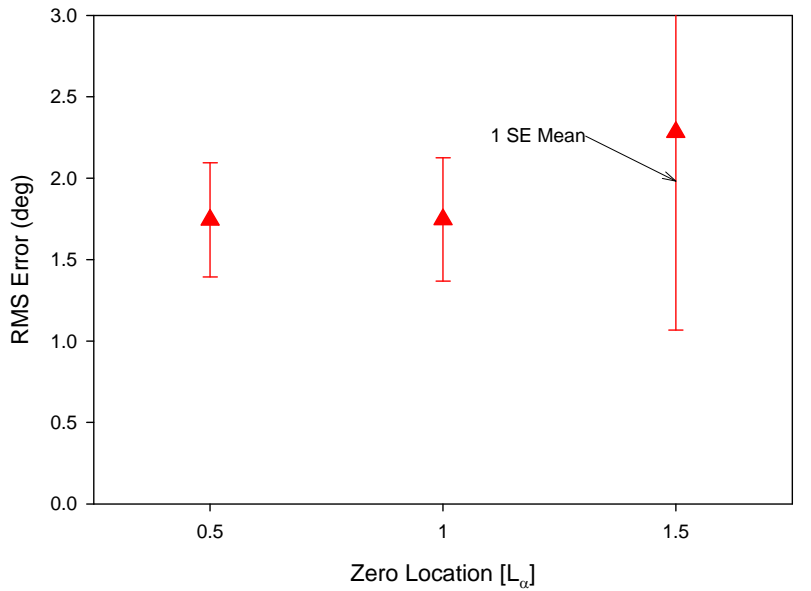

Figure 13. RMS Error by Zero Location

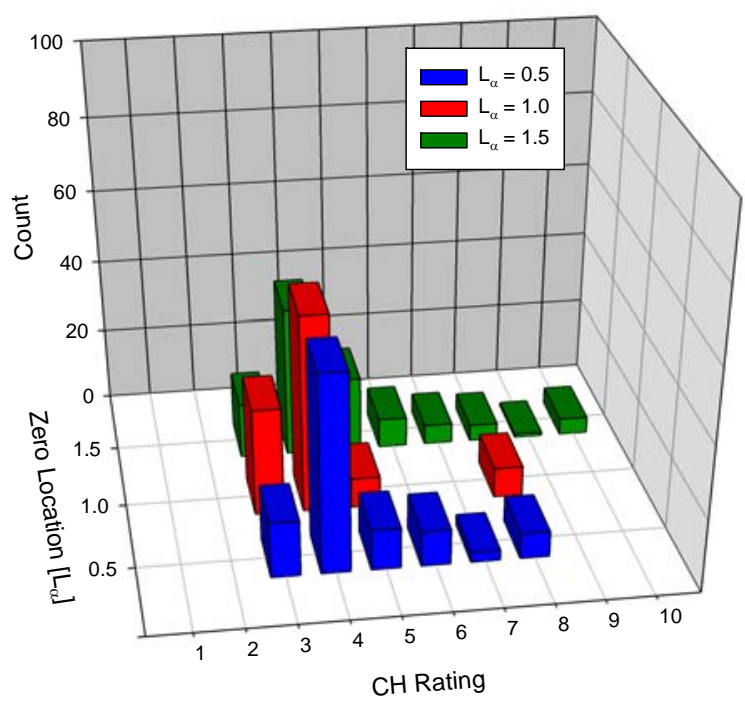

Figure 14. CH Rating Count by Zero Location proportional to $\frac{\omega^{2}}{L_{\alpha}}[13,14]$. In fact, the interaction between the zero location and frequency was significant $\left(F_{(1,207)}=10.001, \mathrm{p} \leq 0.01\right)$ and $\eta^{2}$ was 0.0242 .

\section{Aircraft-Pilot Coupling}

When the system response was slower than the subject desired by either an increasing time delay or decreasing the frequency, especially with a corresponding zero location increase, the RMS error increased. In fact, some subjects mentioned 


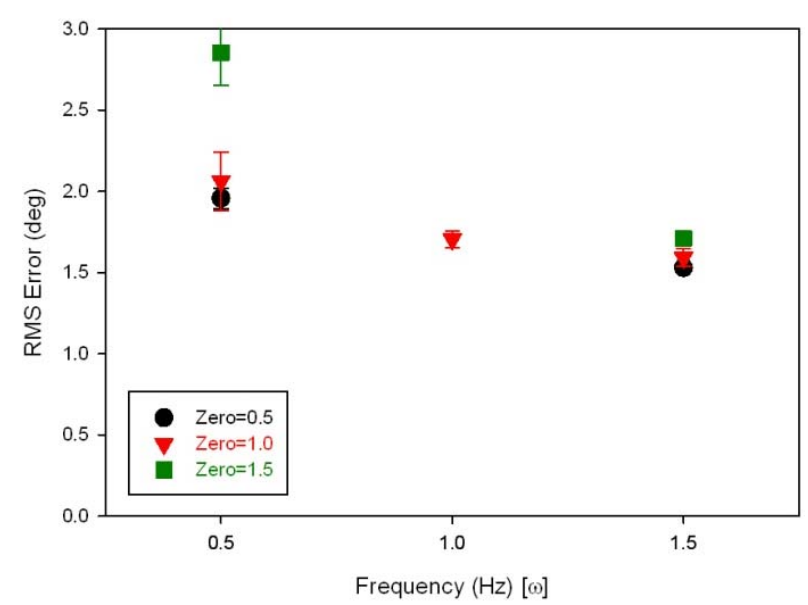

Figure 15. RMS Error by Frequency and Zero

that certain runs were "PIO prone" (Table 3 ). These runs had a frequency of $0.5 \mathrm{~Hz}$ with the zero location farther into the left half plane or a large time delay.

Table 3. PIO Comments

\begin{tabular}{|c|c|c|c|}
\hline $\begin{array}{c}\text { Time } \\
\text { Delay } \\
(\mathbf{m s})[\tau]\end{array}$ & $\begin{array}{c}\text { Frequency } \\
(\mathbf{H z})[\omega]\end{array}$ & $\begin{array}{c}\text { Zero } \\
\text { Location } \\
{\left[\mathbf{L}_{\boldsymbol{\alpha}}\right]}\end{array}$ & $\begin{array}{c}\text { Count of } \\
\text { Subjects } \\
\text { Mentioning } \\
\text { "PIO Prone" }\end{array}$ \\
\hline 0 & 0.5 & 1.0 & 1 \\
\hline 0 & 0.5 & 1.5 & 2 \\
\hline 150 & 0.5 & 0.5 & 4 \\
\hline 150 & 0.5 & 1.5 & 6 \\
\hline
\end{tabular}

Moreover, subjects' $\mathrm{CH}$ ratings became more diverse towards higher $\mathrm{CH}$ ratings as more subjects mentioned "PIO Prone" (Fig. 16).

\section{Pilot Modeling Considerations}

\section{Using System Identification to Determine Hess Simplified Pursuit Model Gain Parameters}

The Hess simplified pursuit pilot model includes two gains, $K_{p}$ and $K_{r}$ and a fixed neuromuscular model to represent a pilot's response to a vehicle [15, $16] . K_{r}$ is the gain that results in a minimum damping ratio of 0.15 and $K_{p}$ is the gain that provides the desired open-loop crossover frequency consistent with the classical McRuer cross-over model $[6,7]$. The pilot-vehicle model is shown in Figure 17 where the vehicle is represent by Equation 1 and $G_{n m}$, the neuromuscular model, is

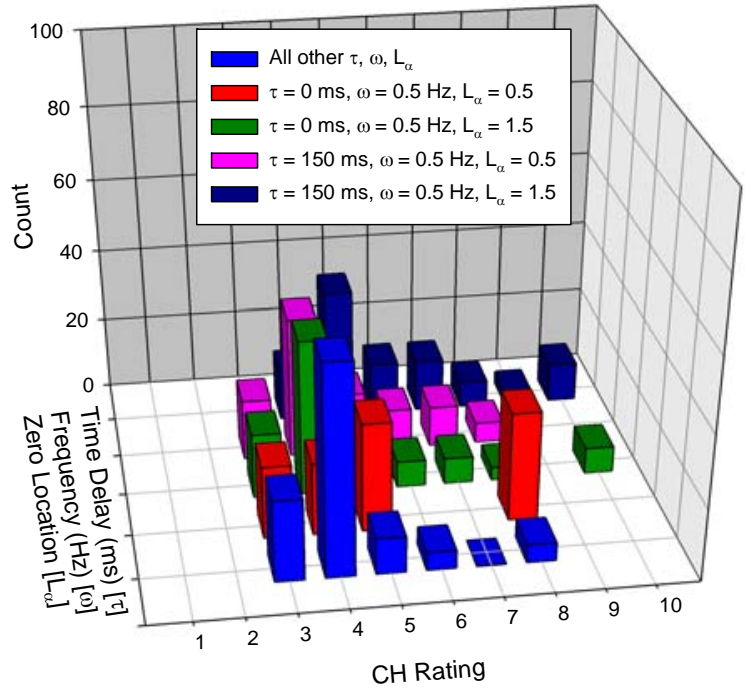

Figure 16. CH Rating Count for PIO Conditions

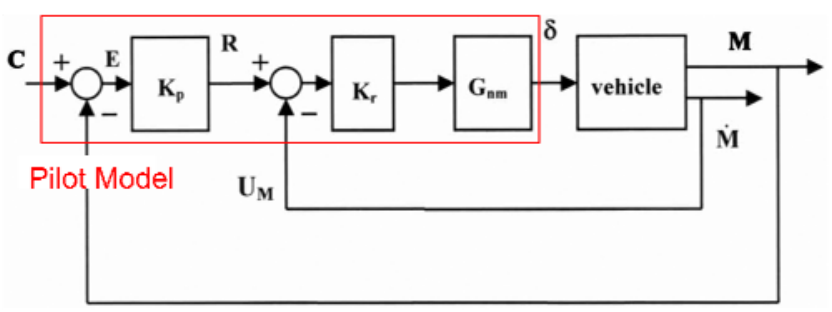

Figure 17. Hess Simplified Pursuit Model

$$
G_{n m}=\frac{10^{2}}{s^{2}+2(0.707) 10 s+10^{2}}
$$

For the purposes of this experiment, input $C$ is commanded pitch attitude $u$, the output $M$ is aircraft pitch attitude $\theta$ and $\dot{M}$ is pitch rate $q$. The above model does not include a time delay. For this analysis, the time delay was incorporated into the Hess simplified pursuit model with a sixth order Padé approximation.

System identification techniques were used to calculate these gains [9] from the recorded data runs. The $K_{p}$ and $K_{r}$ gains vary slightly by pilot and are dependent on the scenarios which had varying system dynamics $\left(f\left(K, L_{\alpha}, \varsigma, \omega, \tau\right)\right)$ (Figs. 18 and 19). The dependency on the subject may be due to learning affects or piloting styles. 


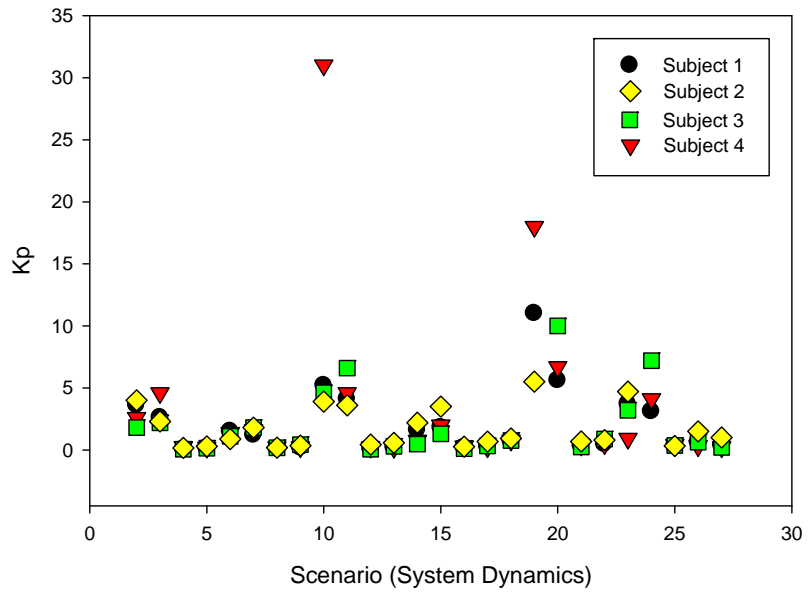

Figure 18. Effects of Scenario and Subject on Kp

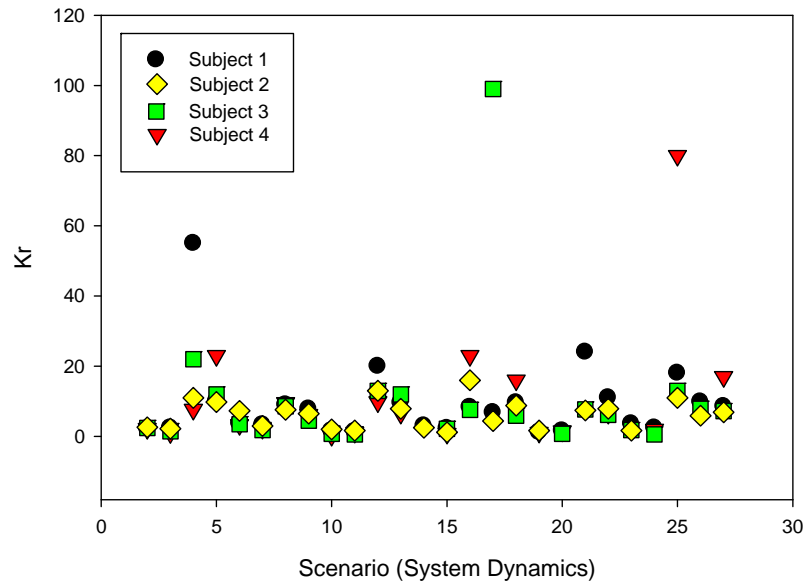

Figure 19. Effects of Scenario and Subject on Kr

Furthermore, within a given subject and constant system dynamics, the calculated $K_{p}$ and $K_{r}$ values using system identification techniques varied by run (Table 4). The values typically settled around an average but there were some outliers. Therefore, the need for real-time system identification is apparent especially if the aircraft dynamics change.

Lastly, the Hess simplified pursuit model was shown to generally emulate the recorded pilot behavior when using the $K_{p}$ and $K_{r}$ values calculated using system identification (Fig. 20 where $K=2, L_{\alpha}=1$, $\zeta=0.7, \omega=1 \mathrm{~Hz}, \tau=75 \mathrm{~ms}$ ). However, the Hess model does not reproduce the higher frequency responses very well.

As expected, these results indicate that the
Table 4. Repetition Effects on Kp and Kr

\begin{tabular}{|c|c|c|}
\hline $\begin{array}{c}\text { Repetition } \\
\left(\boldsymbol{K}=\mathbf{2}, \boldsymbol{L}_{\boldsymbol{\alpha}}=\mathbf{1},\right. \\
\boldsymbol{=} \mathbf{0 . 7}, \boldsymbol{\omega}=\mathbf{1 ~ H z}, \\
\boldsymbol{\tau}=\mathbf{7 5} \mathbf{~ m s})\end{array}$ & $\begin{array}{c}\text { Calculated Kp } \\
\text { Using System } \\
\text { Identification }\end{array}$ & $\begin{array}{c}\text { Calculated Kr } \\
\text { Using System } \\
\text { Identification }\end{array}$ \\
\hline 1 & 0.55 & 9.20 \\
\hline 2 & 0.51 & 6.80 \\
\hline 3 & 0.77 & 8.06 \\
\hline 4 & 0.87 & 6.46 \\
\hline 5 & 0.89 & 5.95 \\
\hline 6 & 1.11 & 7.59 \\
\hline 7 & 0.68 & 6.56 \\
\hline 8 & 0.89 & 8.86 \\
\hline 9 & 0.85 & 7.61 \\
\hline 10 & 0.78 & 5.99 \\
\hline 11 & 1.05 & 7.34 \\
\hline 12 & 0.66 & 8.43 \\
\hline 13 (outlier) & 0.06 & 35.69 \\
\hline $\begin{array}{c}\text { Average } \pm \text { StDev } \\
\text { (for Repetitions }\end{array}$ & $0.80 \pm 0.18$ & $7.40 \pm 1.09$ \\
\hline $1-12)$ & & \\
\hline \multicolumn{2}{|l}{} \\
\hline
\end{tabular}

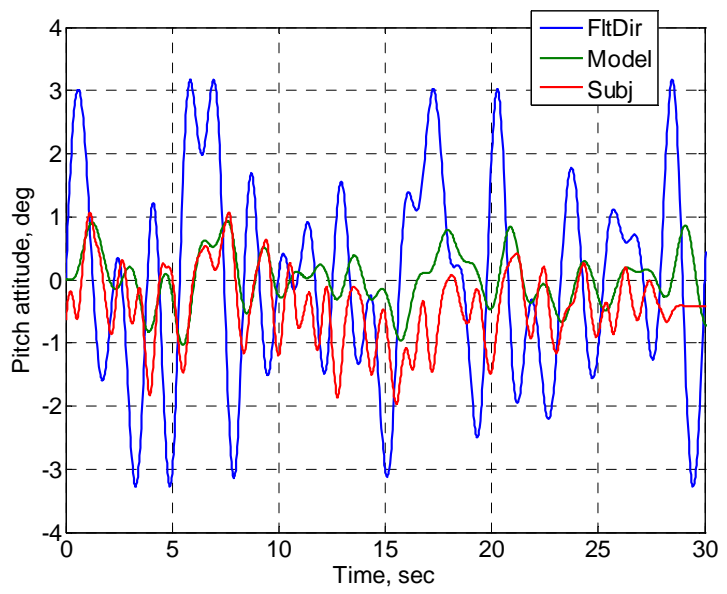

Figure 20. Model vs. Subject Tracking

model changes in pilot control behavior due to the values of $K_{p}$ and $K_{r}$ vary with the aircraft dynamics. The dependence of the pilot model on aircraft dynamics prompts consideration of other techniques that could sudden changes in aircraft dynamics in real-time. The technique considered has been successful in dealing with the changes in aircraft dynamics from the flight control perspective. The ultimate goal is to develop an algorithm that would capture pilot behavior changes due to aircraft dynamic changes without a priori knowledge of how 
these dynamic changes occur. The approach outlined herein is only an initial step.

\section{Estimating Pilot Response with a Simple Gradient Descent Estimator}

A number of different pilot models with a specific structure have been proposed in literature [47, 16]; however, all of these depend on a priori knowledge of aircraft dynamic changes to alter the pilot behavior model. The proposed approach is to assume the pilot can be represented by a system in the linear parameterization form:

$$
y(t)=\Theta^{T} \Phi(x(t))+\varepsilon(t)
$$

where $y(t) \in \square^{m}, x(t) \in \square^{n}$ are the system output and input signals, the $(N \times m)$ - matrix $\Theta \in \square^{N x m}$ contains the unknown parameters to be estimated, $\Phi(x(t)) \in \square^{N}$ (called the "regressor") represents the $N$-dimensional vector of chosen basis functions, and $\varepsilon(t) \in \square^{m}$ denotes the non-parametric uncertainties in the system (such as noise, modeling errors, etc.). Note that in the equation above both $y(t)$ and $\Phi(x(t))$ are known quantities. This implies that (4) is simply a system of linear equations in terms of the unknown $\Theta$ which at time $t$ is estimated by $\hat{\Theta}(t)$. Then based on the latter, one can predict the value of the system output:

$$
\hat{y}(t)=\hat{\Theta}^{T} \Phi(x(t))
$$

where $\hat{y}(t)$ is called the predicted output at time $t$.

Consider a simple gradient estimator to update the predictor output $\hat{y}(t)$. The basic idea in the gradient-based estimation is to update the estimated parameters $\hat{\Theta}(t)$ in such a way that the prediction error $e_{y}=y(t)-\hat{y}(t)$ is reduced. The online implementation of the Gradient Estimator is of the form:

$$
\dot{\hat{\Theta}}(t)=-\Gamma \Phi(x(t))(\hat{y}(t)-y(t))
$$

where $\Gamma=\Gamma^{T}>0$ is a symmetric positive definite matrix called the estimation gain [17].

For pilot model identification, $x(t)$ is the pitch attitude commanded by the flight director while $y(t)$ is the aircraft pitch attitude response to the pilot stick command as the pilot attempts to follow the flight director. The update law for the estimates of the unknown parameters (6) is a function of the tracking error and a regressor chosen a priori. The only assumption about the structure of the pilot model made in this formulation is that it can be represented as linear parameterization.

A sample result for the predicted pilot output using the gradient estimator is given in Figure 21. As can be seen in Figure 21, this initial estimate (ThetaHat_Pilot) follows the pilot's response (Theta_Pilot) fairly well. Furthermore, this method does appear to pick up more of the pilot's high frequency responses.
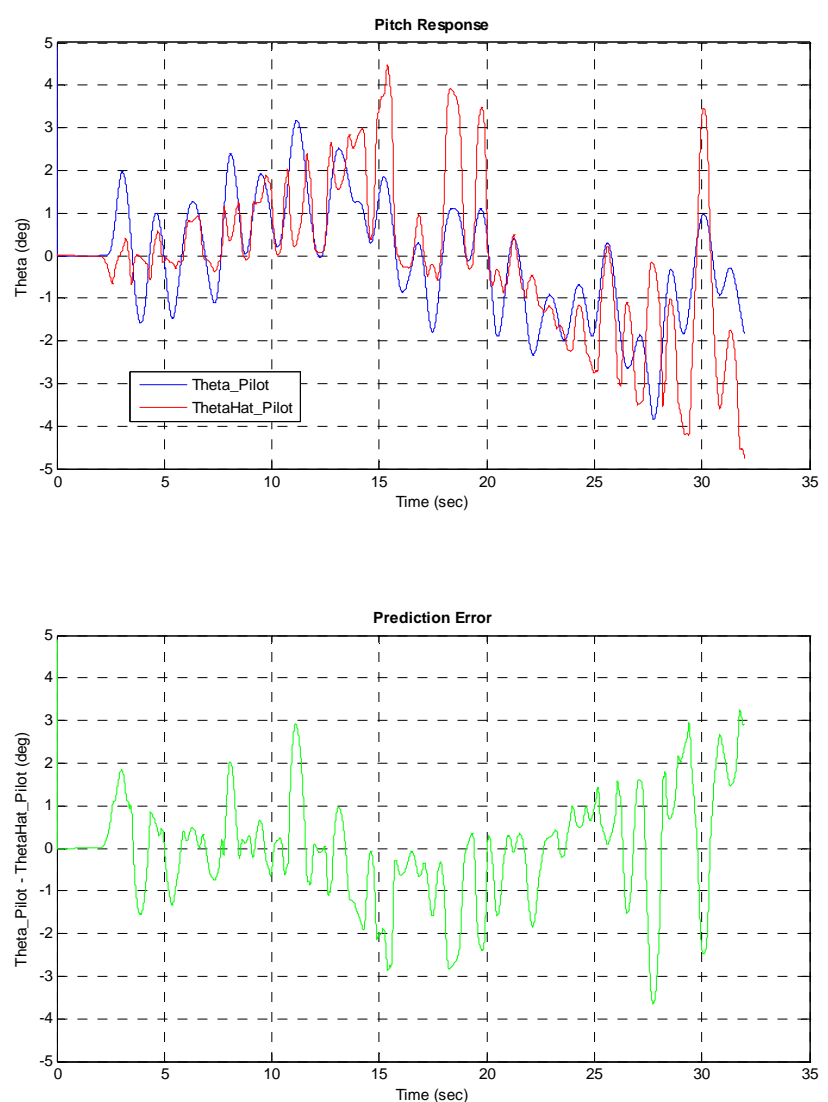

Figure 21. Pilot and Estimator Pitch Response

The parameters of the gradient estimator appear to remain fairly constant from subject to subject with the same pitch dynamics (Fig. 22). However, when the pitch dynamics change within a subject, the current combination of the basis functions $\Phi(x(t))$, which are held constant across all subjects and all dynamics, and the gradient estimator parameter 

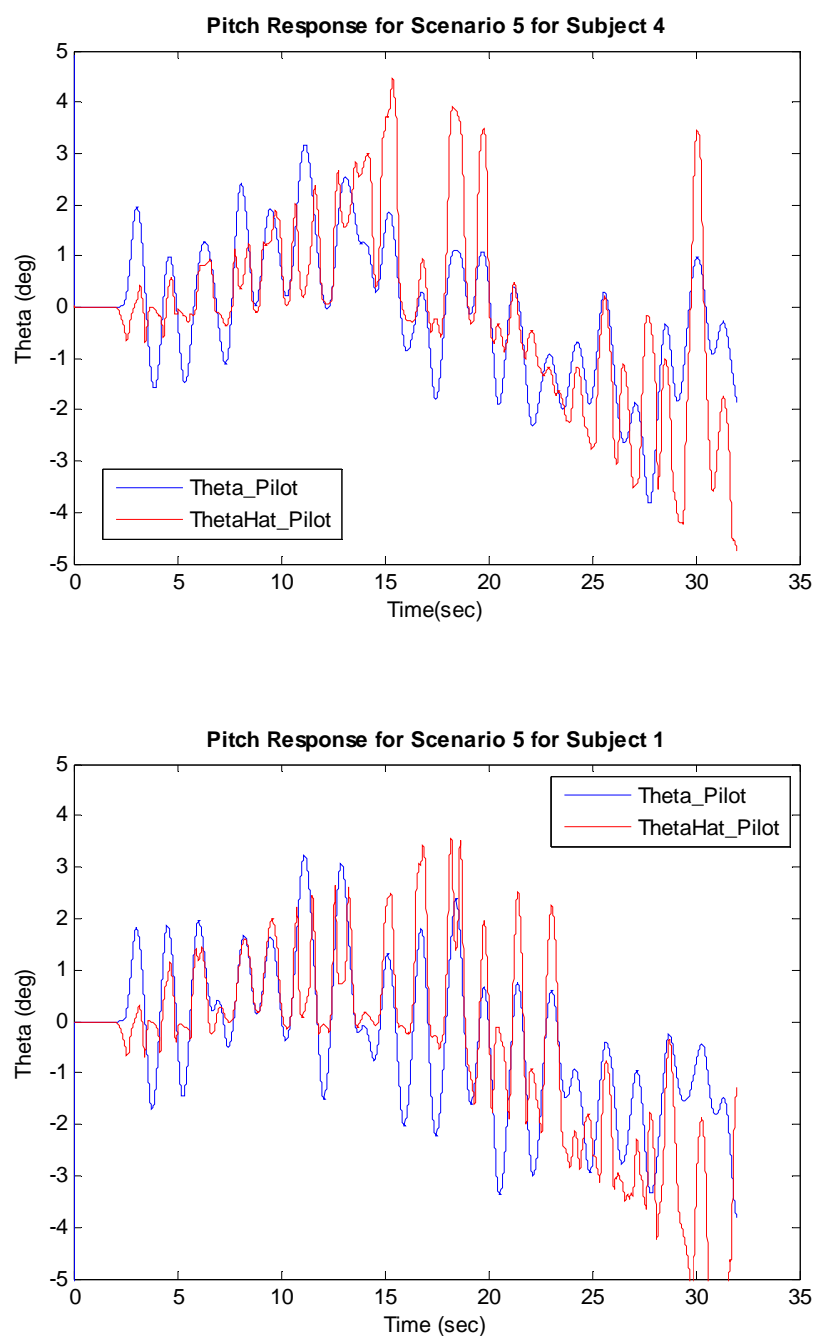

Figure 22. Pitch Response for Different Subjects

update law (6) do not do a good job of covering the range of dynamics. (Fig. 23).

It is clear from Figures 21 and 22 that while the predictor is doing an adequate job in predicting the pilot during some time intervals, i.e., the predictor matches the actual pilot response, but it does a poor job in others. One issue is the estimation methodology as given by the update law (6). The simple gradient descent estimator was used as a proof of concept. Current research is looking at a variety of different approaches to find ones most suitable to predicting the pilot in this general model form.

In this more general model formulation the basis functions vector $\Phi(x(t))$ is expected to cover the range of dynamics and differences among the pilots; the unknown parameter $\hat{\Theta}(t)$ changes in a prescribed manner to accommodate the differences between the
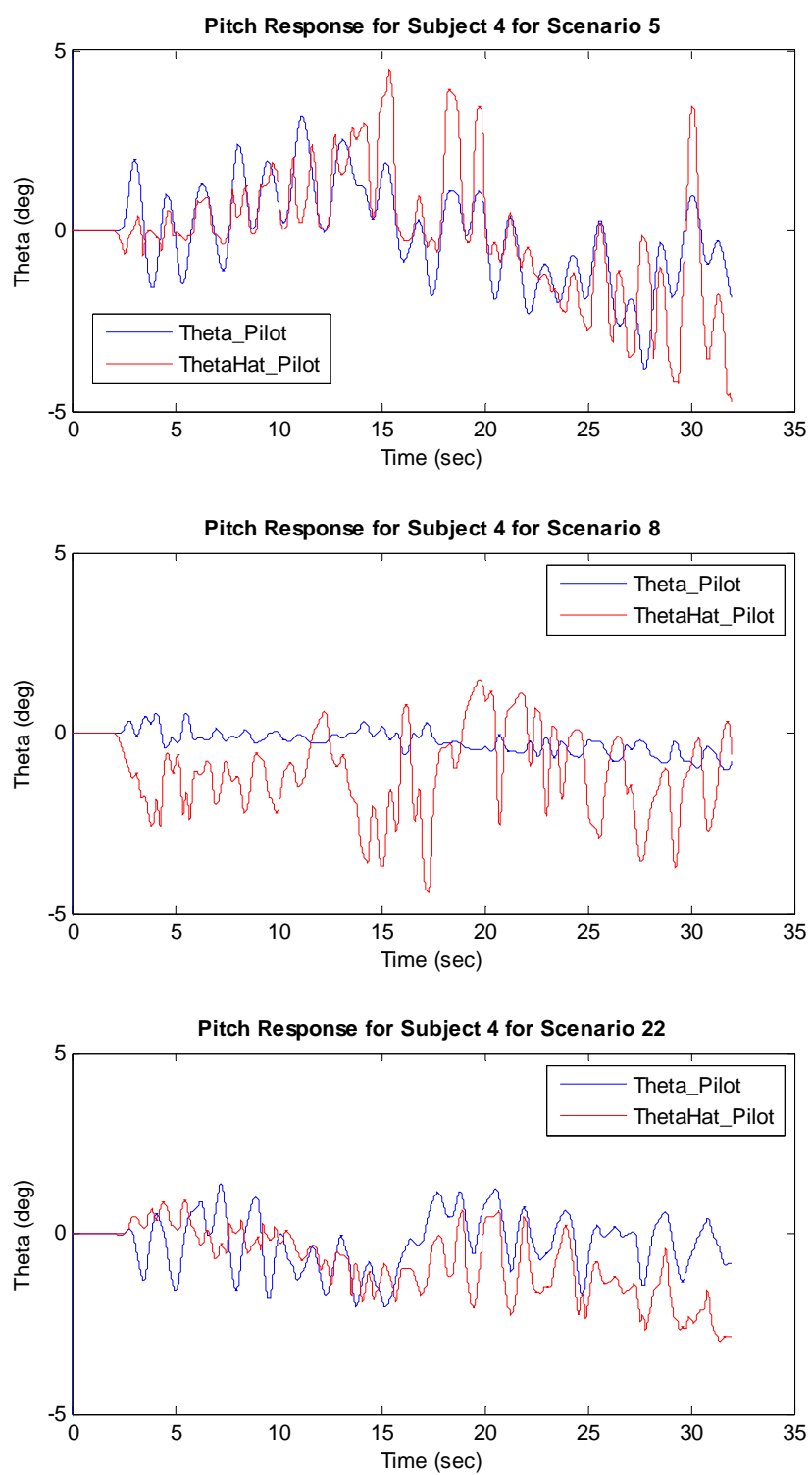

Figure 23. Pitch Response for Different Dynamics

dynamics and pilots based on a specific update law (6). This is different from a fixed structure model where parameters would change with the dynamics, e.g. the Hess model, but would have to be updated manually not based on a specific update expression.

However, it is possible the estimator's parameters $\hat{\Theta}(t)$ may need to be tuned to the current dynamics which may be estimated using real-time system identification techniques [18]. Current research is determining if this is necessary and feasible. If this is feasible, then pilot model would be build on the basis of actual aircraft dynamics. In this case, other pilot model structures $[4-7,16]$ might also be suitable for real-time pilot behavior prediction. 


\section{Conclusions}

An experiment was conducted to quantify the effects of changing dynamics on a subject's ability to track a signal in order to eventually model an adapting pilot to changing aircraft dynamics. The research presented here attempts to identify primary aircraft dynamics variables that influence changes in pilot's response and produce a simplified pilot model that incorporates this relationship. Each run incorporated a different set of second-order aircraft dynamics representing short period transfer function pitch attitude response: damping ratio, frequency, gain, zero location, and time delay.

In the aircraft dynamics, the factors that affected the subjects' ability to track the longitudinal signal the most were the time delay, frequency, and zero location. Besides the subjects' RMS error, subjects' $\mathrm{CH}$ ratings also showed this effect. Furthermore, this increase in RMS error was even more pronounced when looking at the control anticipation parameter and when considering subjects' comments regarding pilot-aircraft coupling.

The Hess simplified pilot model using gains obtained from system identification techniques shows promise in predicting pilots' control behavior but further refinement on the method is needed. Under current consideration is a more general form of the pilot dynamics model borrowed from flight control application dealing with changing aircraft dynamics. Several estimation techniques are being explored to find most suitable approach to predicting the pilot without a priori knowledge of changed aircraft dynamics.

In addition to creating a simplified pilot model to serve as a short-term control action behavioral predictor, the results of the experiment may be utilized in an advisory capacity. Thus, if the aircraft dynamics change such that the system becomes more sluggish and unpredictable to the pilot, an increase in RMS error can be predicted. If a system does in fact encounter these factors (established through real-time parameter identification), advisory information that would aid the pilot in adjusting his piloting approach and changing his expectations of aircraft response can now be provided reliably by the system. A pilot will eventually notice these changes while flying the aircraft but a situation awareness/prediction aid based on the pilot behavior and aircraft dynamics may help tailor pilot's inputs more quickly so that PIO or an upset condition can be avoided.

\section{References}

[1] Hess, R.A. and M.A. Mnich, 1985, Identification of Pilot Dynamics from In-Flight Tracking Data, AIAA Paper 85-1945.

[2] Hess, R.A., 2007, Obtaining Multi-Loop PursuitControl Pilot Models From Computer Simulation, AIAA-2007-247.

[3] Bailey, R.E. and D.J. Mook, 1992, Pilot Control Identification using Minimum Model Error Identification, AIAA 92-1121.

[4] Davidson, J.B. and D.K. Schmidt, 1992, Modified Optimal Control Pilot Model for Computer-Aided Design and Analysis, in NASA TM-4384, NASA.

[5] Doman, D.B. and M.R. Anderson, 2000, A FixedOrder Optimal Control Model of Human Operator Response, Automatica, 36(3), pp. 409-418.

[6] McRuer, D.T. and H.R. Jex, 1967, A Review of Quasi-Linear Pilot Models, IEEE Transactions on Human Factors in Electronics, HFE8(3), pp. 231-249.

[7] McRuer, D.T. and E.S. Krendel, 1974, Mathematical Models of Human Pilot Behavior, AGARD-AG-188.

[8] Morelli, E.A., 2008, Flight Test Experiment Design for Characterizing Stability and Control of Hypersonic Vehicles, in AIAA-2008-1682, U.S. Air Force T\&E Days, Los Angelos, CA.

[9] Klein, V. and E.A. Morelli, 2006, Aircraft System Identification Theory and Practice, AIAA.

[10] Cooper, G.E. and R.P. Harper, 1969, The Use of Pilot Rating in the Evaluation of Aircraft Handling Qualities, NASA TN D-5153.

[11] Harper, R.P. and G.E. Cooper, 1986, Handling Qualities and Pilot Evaluation (Wright Brothers Lecture in Aeronautics), Journal of Guidance, Control, and Dynamics, 9(6), pp. 515-529.

[12] Trujillo, A.C., 2009, Paper to Electronic Questionnaires: Effects on Structured Questionnaire Forms, in HCI International 2009, J.A. Jacko, Editor, San Diego, CA, Springer-Verlag Berlin Heidelberg, pp. 362-371. 
[13] Hodgkinson, J., 1999, Aircraft Handling Qualities. AIAA Education Series, ed. J.S. Przemieniecki, Blackwell Science, Ltd., pp. 246.

[14] Etkin, B., 1972, Dynamics of Atmospheric Flight, New York, John Wiley \& Sons, Inc., pp. 579.

[15] Hess, R.A., 2006, Simplified approach for modeling pilot pursuit control behaviour in multiloop flight control tasks, Proceedings of the Institution fo Mechanical Engineers, Vol. 220 Part G: Journal of Aerospace Engineering, pp. 85-102.

[16] Hess, R.A., 2008, Modeling the Adaptive Human Pilot Accommodating Sudden Changes in Vehicle Dynamics, Davis, CA, Dept. of Mechanical and Aeronautical Engineering, University of California, pp. 13.

[17] Lavretsky, E., 2008, CDS 270 - System Identification Class Notes, Spring 2008, California Institute of Technology.

[18] Morelli, E.A., 2011, Aircraft System Identification Briefing to NESC Technical Displine Teams, in NESC Technical Interchange, Green Bay, WI.

\section{Acknowledgements}

The authors gratefully acknowledge the significant contributions of the many members of the research team. Deserving of credit are Dr. Gene Morelli and Chris Hartman of ATK Space Systems who did the system identification of the piloting dynamics. Also deserving of credit is Lucas Hempley of Lockheed Martin for his programming of the experiment.

\section{Disclaimer}

The use of trademarks or names of manufacturers in the report is for accurate reporting and does not constitute an official endorsement, either expressed or implied, of such products or manufacturers by the National Aeronautics and Space Administration.

30th Digital Avionics Systems Conference

October 16-20, 2011 